\title{
LETTER \\ Outage Analysis of Cognitive Multihop Networks under Interference Constraints
}

\author{
Vo Nguyen Quoc BAO ${ }^{\dagger \mathrm{a})}$ and Trung Quang DUONG ${ }^{\dagger \dagger \mathrm{b})}$, Members
}

\begin{abstract}
SUMMARY In this letter, we consider a cognitive radio based multihop network under the spectrum sharing underlay paradigm. By taking into account the interference constraints, we present an exact closed-form expression for outage probability, which is valid for the whole signal-to-noise ratio regime. In addition, some numerical examples of interest that study the effect of the number of hops and/or the interferer threshold on primary users are illustrated and discussed. Numerical results show that multihop systems still offer a considerable gain as compared to direct transmission under the same limit of interference.

key words: decode-and-forward, underlay, multihop communication, assisted relay networks, cognitive radio
\end{abstract}

\section{Introduction}

Multihop relaying communication - the data transmission technique between a source and a destination that is based on a certain number of intermediate relay nodes - has been a long lasting research focus worldwide [1], [2]. Recently, the concept of relaying communication has been introduced for cognitive radio networks and appears to be a promising solution to achieve stable connectivity among cognitive nodes. With the help of secondary users (SUs) acting as forwarders, the communication between the secondary source and the secondary destination is considerably increased, resulting in a certain quality of service (QoS) improvement for secondary networks. On the other hand, thanks to multihop relaying, secondary nodes are able to use lower transmission power without imposing harmful interference on primary users (PUs) [3]-[5].

Recent literature has discussed multihop relaying networks in cognitive radio from various perspectives, (see, e.g., [4]-[9]). In [4], the concept of ad-hoc cognitive radio was used for realizing multihop frequency-sharing networks to expand network service areas. In [5], the approximate outage probability and diversity order of repetition-based cognitive relay networks were studied, in which cognitive relays operate in unused frequency bands only. For a similar scenario, the closed-form expression of outage probability for cognitive opportunistic networks was derived, showing that the inability of relays to sense unoccupied spectrum

\footnotetext{
Manuscript received June 7, 2011.

Manuscript revised October 11, 2011.

${ }^{\dagger}$ The author is with the Posts and Telecommunications Institute of Technology, Ho Chi Minh City, Vietnam.

${ }^{\dagger}$ The author is with the Blekinge Institute of Technology, SE37179 Karlskrona, Sweden.

a) E-mail: baovnq@ @ ptithcm.edu.vn

b) E-mail: quang.trung.duong@bth.se

DOI: 10.1587/transcom.E95.B.1019
}

can cause a larger drop in the network performance, especially in high signal-to-noise ratio (SNR) regime [6]. Considering the maximum allowable interference level at the PU, the performance of relay-assisted cognitive radio networks in terms of outage probability was provided in [7]. Taking into account the presence of a single or multiple active primary links, two distributed transmit power allocation schemes were proposed in [8] with an aim to optimize the performance of the cognitive radio systems. Very recently, the optimal power allocation schemes for secondary dual-hop networks under limited interference to PUs have been developed in [9] offering an improvement of the system throughput by over $50 \%$.

In this work, we investigate the advantages of deploying multihop transmission for cognitive radio networks under interference constraints. Assuming that each hop experiences independent, but not necessarily identically distributed Rayleigh fading, the closed-form expression for outage probability of cognitive multihop decode-andforward (DF) relay networks is derived. Some representative numerical results are presented and discussed. Based on these results, new and interesting questions arising in cognitive multi-hop networks under interference constraints are answered. What is the effect of the number of SUs on the system performance? How many hops should be used for a particular setting condition? And what is the performance of the networks when the location of the PU is changed? Answers for those questions are not straightforward, and this paper is further dedicated to shedding light on some of the issues that arise in such networks.

\section{System Model}

We consider a multihop spectrum sharing communication system where SUs can operate in the spectrum band licensed by a PU based on cognitive radio technology. The transmission between the secondary source $\left(\mathrm{CR}_{1}\right)$ and the secondary destination $\left(\mathrm{CR}_{K+1}\right)$ takes place in orthogonal timeslots with the help of $K-1$ cognitive relays over $K$ hops transmission. Equipped with the DF relaying protocol, intermediate users always fully decode the received signal from their preceding node, and then forward the re-encoded version to their respective successor node.

Consider the $k$-th hop, the channel gains of the link from the $k$-th secondary transmitter to the next secondary receiver and to the primary receiver are denoted by $h_{D, k}$ and $h_{I, k}$, respectively. In the underlay approach, the $k$-th $\mathrm{SU}$ is 
permitted to use the licensed band of the PU as long as it does not harm the PU communication. In particular, the transmission power of secondary node $k$ must satisfy not only the interference constrains of the primary node, i.e., $P_{k}\left|h_{D, k}\right|^{2} \leq I$, where $I$ defines the maximum tolerable interference level of the PU, but also the maximum transmission power, i.e., $P_{k} \leq P_{m}$, given by $P_{k}=\min \left\{\frac{I}{\left|h_{l, k}\right|^{2}}, P_{m}\right\}$, where $P_{m}$ is the maximum transmission power. The instantaneous SNR of the source-to-destination link under interference constraint is given by [8], [10]

$$
\gamma_{k}=\min \left\{I / N_{0} \frac{\gamma_{D, k}}{\gamma_{I, k}}, \gamma_{D, k}\right\},
$$

where $N_{0}$ denotes the variance of additive white Gaussian noise at all receiving terminals, $\gamma_{D, k}=P_{m}\left|h_{D, k}\right|^{2} / N_{0}$, and $\gamma_{I, k}=P_{m}\left|h_{I, k}\right|^{2} / N_{0}$. Under Rayleigh fading channels, it is obvious that $\gamma_{D, k}$ and $\gamma_{I, k}$ are exponentially distributed random variables with parameters $\bar{\gamma}_{D, k}=P_{m} \lambda_{D, k} / N_{0}$ and $\bar{\gamma}_{I, k}=P_{m} \lambda_{I, k} / N_{0}$ [11], respectively, where $\lambda_{D, k}=E\left\{\left|h_{D, k}\right|^{2}\right\}$ and $\lambda_{I, k}=E\left\{\left|h_{I, k}\right|^{2}\right\}$. Here, $E\{$. $\}$ denotes the expectation operator. We are now in a position to derive the statistics of $\gamma_{k}$. We first present the cumulative distribution function (CDF) of $\gamma_{k}$, which will be used for the derivation of the performance metrics.

Theorem 1. The CDF of the SNR for each hop under interference constraint is given by

$$
F_{\gamma_{k}}(\gamma)=1-e^{-\frac{\gamma}{\bar{\gamma}_{D, k}}}+\frac{\gamma \bar{\gamma}_{D, k} e^{-\left(\frac{\gamma}{\bar{\gamma}_{D, k}}+\frac{I I N_{0}}{\bar{\gamma}_{I, k}}\right)}}{\gamma / \bar{\gamma}_{D, k}+I / N_{0} / \bar{\gamma}_{I, k}} .
$$

Proof. From (1), we have

$$
\begin{aligned}
F_{\gamma_{k}}(\gamma)= & \operatorname{Pr}\left(\gamma_{I, k} \leq I / N_{0}, \gamma_{D, k} \leq \gamma\right) \\
& +\operatorname{Pr}\left(\gamma_{I, k}>I / N_{0}, \gamma_{D, k} \leq \gamma \frac{\gamma_{I, k}}{I / N_{0}}\right) .
\end{aligned}
$$

Figure 1(a) and Fig. 1(b) show the areas corresponding to the first and second term, respectively, in the sum of (3). Integrating over these two regions, we get

$$
\begin{aligned}
& F_{\gamma_{k}}(\gamma)=\int_{0}^{I / N_{0}} f_{\gamma_{I, k}}\left(\gamma_{I, k}\right) d \gamma_{I, k} \int_{0}^{\gamma} f_{\gamma_{D, k}}\left(\gamma_{D, k}\right) d \gamma_{D, k} \\
& +\int_{I / N_{0}}^{+\infty} f_{\gamma_{I, k}}\left(\gamma_{I, k}\right) d \gamma_{I, k} \int_{0}^{\gamma \gamma_{I, k} / I / N_{0}} f_{\gamma_{D, k}}\left(\gamma_{D, k}\right) d \gamma_{D, k} .
\end{aligned}
$$

(a)
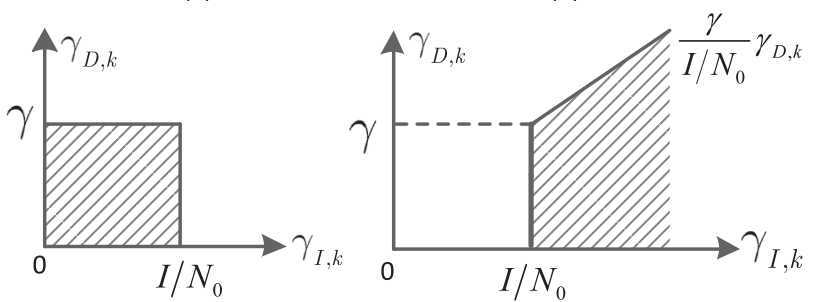

Fig. 1 The areas corresponding to the first and second term in the sum of (3).
By solving (4), we can achieve (2), which completes the proof.

\section{Outage Probability}

In this section, we evaluate the outage probability of mutihop DF networks by taking into account the maximum interference power constraints. As defined in [1], the achievable end-to-end data rate of multihop communication is the minimum rate among all hops, namely, $R=\frac{1}{K} \min _{k=\{1, \ldots, \mathrm{K}\}} R_{k}$. Here $R_{k}=\log _{2}\left(1+\gamma_{k}\right)$ and the ratio of $1 / K$ represents the fact that data transmission between the secondary source and the secondary destination takes places in $K$ consecutive time-slots. Outage event occurs when the mutual information of the secondary source-destination pair falls below a specified threshold, i.e., the desired spectral efficiency, $R_{T}$. Therefore, the outage probability is given by

$$
\mathrm{OP}=\operatorname{Pr}\left(\frac{1}{K} \min _{k=1, \ldots, \mathrm{K}} R_{k} \leq R_{T}\right) .
$$

By introducing $\gamma_{t h}=2^{K R_{T}}-1$ and substituting (2) into (5), we get

$$
\mathrm{OP}=1-\prod_{k=1}^{K}\left[e^{-\frac{\gamma_{t h}}{\bar{\gamma}_{D, k}}}-\frac{\gamma_{t h} / \bar{\gamma}_{D, k} e^{-\left(\frac{\gamma_{t h}}{\bar{\gamma}_{D, k}}+\frac{I N N_{0}}{\bar{\gamma}_{I, k}}\right)}}{\gamma_{t h} / \bar{\gamma}_{D, k}+I / N_{0} / \bar{\gamma}_{I, k}}\right] .
$$

\section{Numerical Results and Discussion}

We consider a linear multihop network (MHN) where all nodes are located across a straight line. Specifically, the secondary source, the secondary destination, and the primary node are located at coordinates $(0,0),(1,0)$ and $(0.5,1)$, respectively. Without loss of generality, all secondary nodes are equidistant from each other, i.e., $d_{\mathrm{CR}_{k}, \mathrm{CR}_{k+1}}=1 / K$ where $d_{\mathrm{CR}_{k}, \mathrm{CR}_{k+1}}$ is the distance from secondary node $k$ to secondary node $k+1$ and $d_{\mathrm{CR}_{k} \text {,PU }}$ is the distance between secondary node $k$ and the primary node. Including path loss and fading, the average channel power due to transmission between node $\mathrm{A}$ and $\mathrm{B}$ is modeled as $\lambda_{\mathrm{A}, \mathrm{B}}=d_{\mathrm{A}, \mathrm{B}}^{-\eta}$ with $\mathrm{A} \in\left\{\mathrm{CR}_{1}, \ldots, \mathrm{CR}_{K}\right\}$ and $\mathrm{B} \in\left\{\mathrm{PU}, \mathrm{CR}_{2}, \ldots, \mathrm{CR}_{K+1}\right\}$. And $\eta$ denotes the path loss exponent and its typical value is in the range of 2 (free space) to 6 (dense urban areas) [3]. For simplicity, equal power allocation in which transmitting nodes use the same amount of power is considered, i.e., $P_{k}=P / K$, where $P$ denotes the transmitting power of the secondary source in direct transmission (DT) mode.

Figure 2 shows the end-to-end outage probability achieved of multihop spectrum-sharing networks under interference constraints. It is shown that the system outage probability grows as the transmitting power of secondary nodes increases. However, it is saturated at a certain level of average SNRs, determined by the interference threshold. The results also confirm the benefit of MHNs under interference constraints as compared to the DT under the same 


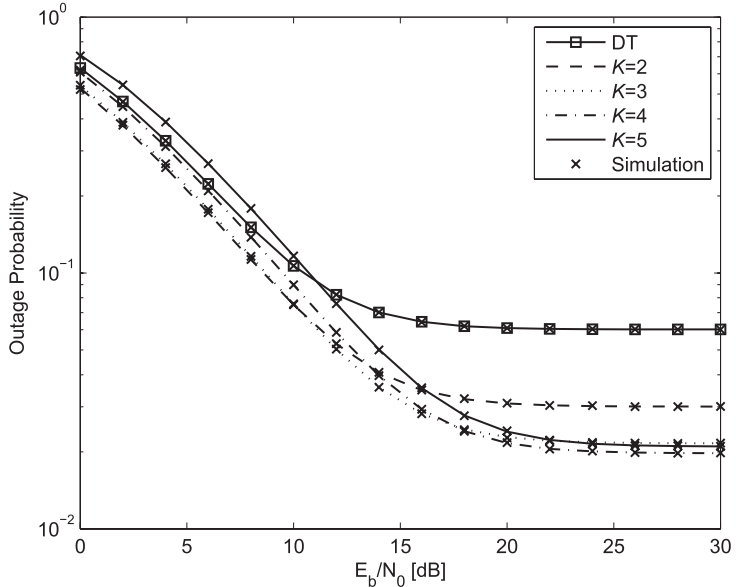

Fig. 2 Outage probability versus average SNRs, $\eta=4, I / N_{0}=10 \mathrm{~dB}$, $R=1$.

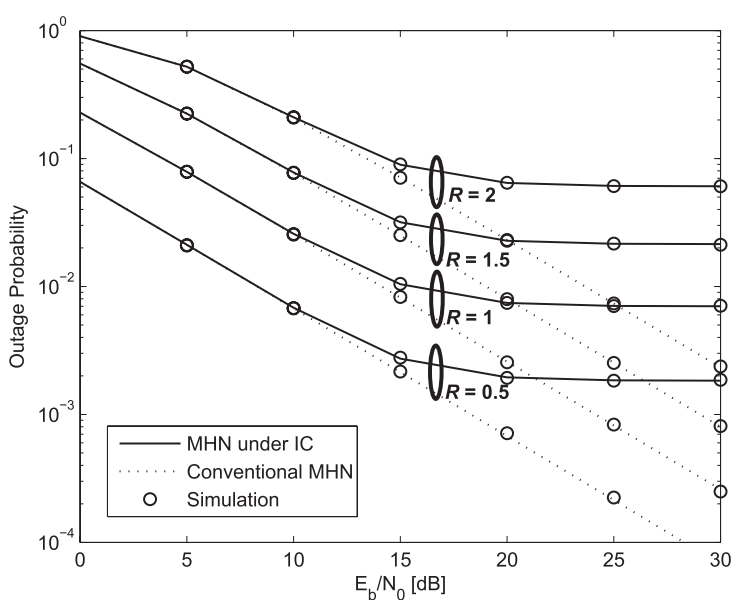

Fig. 3 Effects of $R$ on the system outage probability, $\eta=5, K=3$, and $I / N_{0}=10 \mathrm{~dB}$

condition of interference. For example, except for the case of the network with five hops, MHNs always outperform the DT in low SNR regime.

Figure 3 shows the outage probability as a function of the normalized average SNR for different $R$. In addition, the outage performance of MHNs with no inference constraints is plotted for comparison with the same network topologies and channel settings. Note that for both networks, the increase in $R$ decrease the performance in all SNR range and their outage performance is almost the same for low SNR regime. At high SNRs regime, different from the performance of conventional MHNs with no inference constraints, the performance of multihop spectrum sharing networks reaches to an irreducible outage probability. Intuitively, the amount of this irreducible outage probability can be related to the interference threshold as well as the average channel power of the interference links.

Figure 4 shows the outage probability versus $I$ for different values of $E_{b} / N_{0}$. In particular, we vary the interference threshold while the transmitting power is fixed. An

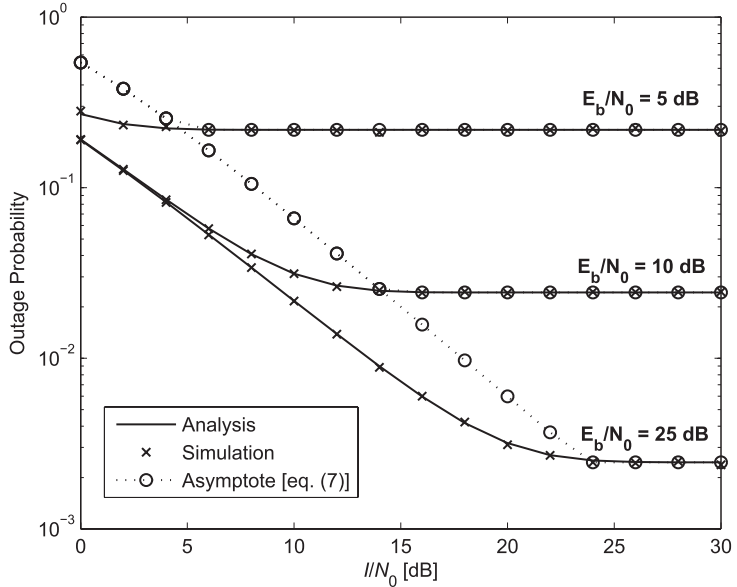

Fig. 4 Outage probability versus interference threshold $(I), \eta=4, R=1$, and $K=3$.

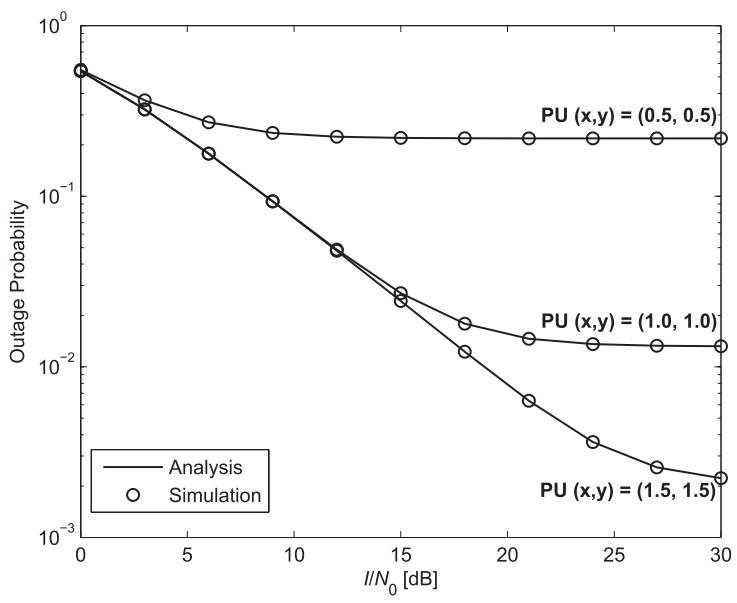

Fig. 5 Effects of the primary node locations on the outage probability, $\eta=4, R=1$, and $K=3$.

interesting observation drawn from Fig. 4 is that when the average SNR is greater than $I / N_{0}$, the system performance converges to a constant. In general, from Fig. 4 and other numerical experiments omitted here due to space limit, we observe that such constant equals the outage probability of the conventional MHNs in which the transmitting power is adjusted as

$$
\min \left(P_{m}, I / \max _{k} \lambda_{\mathrm{CR}_{k}, \mathrm{PU}}\right) .
$$

In Fig. 5, we examine the effect of locations of the PU on the end-to-end outage performance. Observing the results in Fig. 5, we can see that the system performance improves when the primary node is located farther away from the secondary relaying nodes. Beside, simulation results are in excellent agreement with the analysis results.

The effect of path loss exponent on the system outage probability is examined in Fig. 6 . We can see that for the same network topology the larger the value of $\eta$, the greater the attenuation of transmitted signals. Therefore, the outage probability decreases as $\eta$ increases, as expected. Also, in 


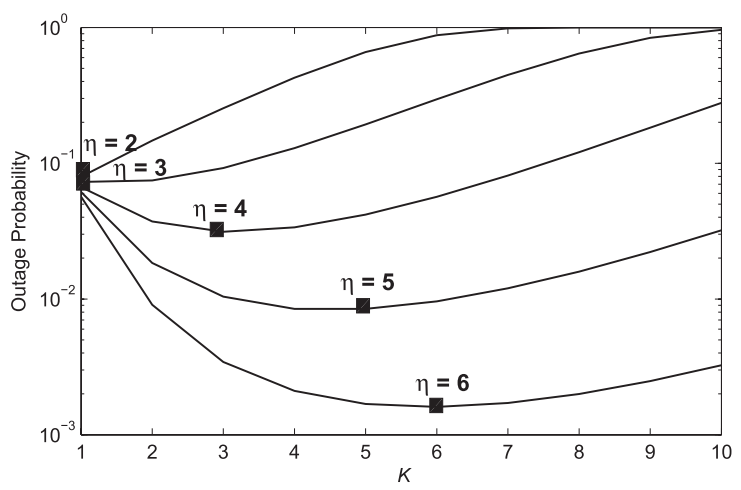

Fig. 6 Effects of path loss exponent $(\eta)$ on the outage probability, $R=1$, $I / N_{0}=10 \mathrm{~dB}$ and $E_{b} / N_{0}=15 \mathrm{~dB}$.

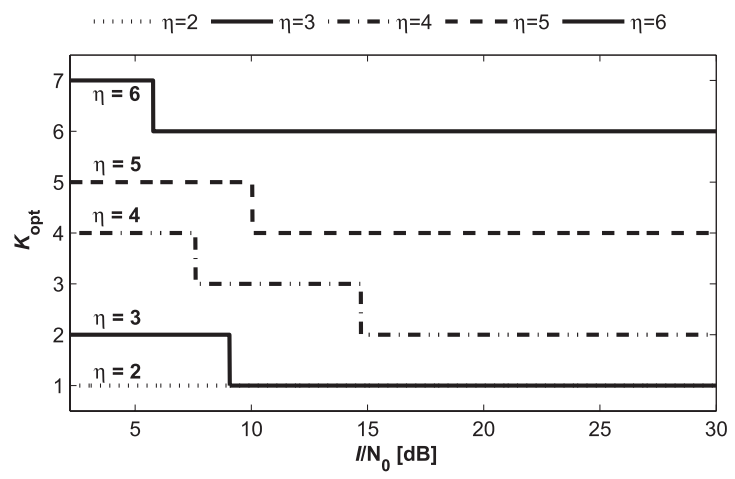

Fig. 7 Effects of interference levels on the optimal number of hops.

Fig. 6, the most outage efficient linear network can be found. Mathematically speaking, we have $K_{\text {opt }}=\arg \min _{K} \mathrm{OP}$. However, with the current form of (6), $K_{o p t}$ is difficult (if not impossible) to obtain in closed-form solution. Consequently, simulation is used here to find the optimal number of hops. As can be clearly seen in Fig. 6, the optimal number of hops is increased as the path loss exponent increases, i.e., $K_{\text {opt }}=\{1,1,3,5,6\}$ for $\eta=\{2,3,4,5,6\}$, correspondingly. To this end, we can conclude that in MHNs, the faster the attenuation of the fading channel gain with distance, the greater the number of secondary nodes is expected to compensate for the path loss.

The effect of interference levels on the optimal number of hops is investigated in Fig. 7. From the figure, we can see that $K_{o p t}$ decreases with an increase of interference power, $I$. This is because the transmission power of secondary nodes decreases when $K_{\text {opt }}$ increases, resulting in lower interference power at the PU.

\section{Conclusions}

In this paper, outage performance of cognitive MHNs un- der interference constraints has been investigated. The exact expression of outage probability has been found and confirmed by simulation results, showing that interference regulation for licensed users changes the characteristics of MHNs previously known in non-spectrum sharing systems. While this work only studies MHNs under interference constraints from a physical-layer point of view, future work must consider the implication of multihop data transmission on higher layers.

\section{Acknowledgment}

This research was supported by the Vietnam's National Foundation for Science and Technology Development (NAFOSTED) (No. 102.99-2010.10).

\section{References}

[1] M.O. Hasna and M.S. Alouini, "Outage probability of multihop transmission over nakagami fading channels," IEEE Commun. Lett., vol.7, no.5, pp.216-218, 2003.

[2] V.N.Q. Bao and H.Y. Kong, "A simple performance approximation for multi-hop decode-and-forward relaying over rayleigh fading channels," IEICE Trans. Commun., vol.E92-B, no.11, pp.35243527, Nov. 2009.

[3] A. Goldsmith, S.A. Jafar, I. Maric, and S. Srinivasa, "Breaking spectrum gridlock with cognitive radios: An information theoretic perspective," Proc. IEEE, vol.97, no.5, pp.894-914, 2009.

[4] T. Fujii and Y. Suzuki, "Ad-hoc cognitive radio - Development to frequency sharing system by using multi-hop network," IEEE DySPAN 2005, pp.589-592, Baltimore, USA, 2005.

[5] L. Kyounghwan and A. Yener, "Outage performance of cognitive wireless relay networks," IEEE GLOBECOM '06., pp.1-5, San Francisco, CA, 2006.

[6] H.A. Suraweera, P.J. Smith, and N.A. Surobhi, "Exact outage probability of cooperative diversity with opportunistic spectrum access," IEEE ICC Workshops '08, pp.79-84, Beijing, China, 2006.

[7] Y. Guo, G. Kang, N. Zhang, W. Zhou, and P. Zhang, "Outage performance of relay-assisted cognitive-radio system under spectrumsharing constraints," Electron. Lett., vol.46, no.2, pp.182-184, 2010.

[8] J. Mietzner, L. Lampe, and R. Schober, "Distributed transmit power allocation for multihop cognitive-radio systems," IEEE Trans. Wireless Commun., vol.8, no.10, pp.5187-5201, 2009.

[9] L. Li, X. Zhou, H. Xu, G.Y. Li, D. Wang, and A. Soong, "Simplified relay selection and power allocation in cooperative cognitive radio systems," IEEE Trans. Wireless Commun., vol.10, no.1, pp.33-36, 2011.

[10] B. Tae Won, C. Wan, J. Bang Chul, and S. Dan Keun, "Multi-user diversity in a spectrum sharing system," IEEE Trans. Wireless Commun., vol.8, no.1, pp.102-106, 2009.

[11] J.G. Proakis, Digital communications, 4th ed., McGraw-Hill, Boston, 2001. 